\title{
РЕЗУЛЬТАТЫ КОЛИЧЕСТВЕННОЙ СТРАТИГРАФИЧЕСКОЙ КОРРЕЛЯЦИИ КЕЛЛОВЕЯ ЮГО-ЗАПАДНОЙ ПРИБАЛТИКИ
}

В рамках исследований по проекту № 148 Международной пропраммы геологической корреляции «Техника количественной стратиграфической корреляции» проверялась работоспособность принятого советской рабочей группой алгоритма составления порядковых шкал на основе распространения фораминифер келловея Юго-Западной Прибалтики. Из-за малого объема проверяемого материала соответствующая его обработка была проведена вручную. В целях ознакомления с выбранным алгоритмом и с возможностями его использования ниже приведены принципиальнь:е процедуры сбработки материала по нему.

Нсходный материал. Анализировали распределение 26 видов фораминифер в 12 разрезах келловейского яруса (средний и верхний подъярусы) Юго-Западной Прибалтики (табл. 1).

Постановка задачи. Среди существующих алгоритмов спратиграфической корреляции (Brower, Millendorf, 1978) имеются такие, которые направлены на выяснение временных соотношений между разрезами (Рубель, 1976; Rubel, 1978; Pak, в печати). Согласно им, оперва составляется единая для всех коррелируемых разрезов шкала времени из наблюдаемых там палеонтологических событий. Затем полученная таким образом порядковая шкала идентифицируется в коррелируемых разрезах в целях выяснения отношений «выше - моложе» между определенными интервалами разрезов. Так как подобные алгоритмы моделируют логический аппарат биостратиграфических обработок вообще (Олейников, Паевская, в печати), то можно ожидать весьма значительного совпадения результатов по выбранному алгоритму с таковыми, полученными традиционным путем.

Процедуры. 1. Установим интервалы распространения видов (или любых таксонов) по их появлению и исчезновению в каждом разрезе.

2. Выясним взаимоположение указанных интервалов по каждому разрезу. После этого колонку с глубинами, как и повторяющиеся строки, можно опустить. Тогда, например, из табл. 1 по скважине Лесной-50 получим следующее относительное распространение видов:

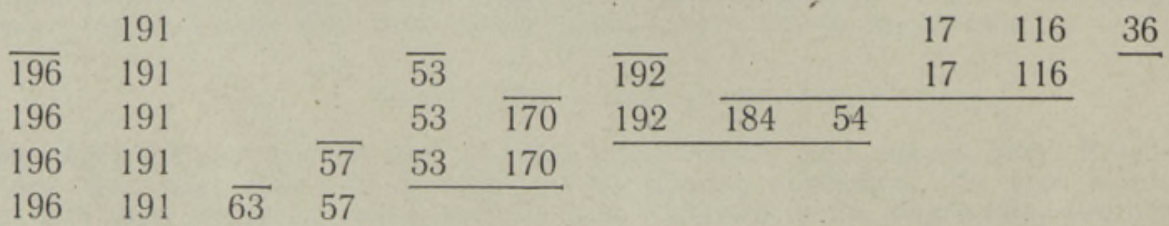

Таким путем легко выявляются относительно друг друга последовательные и сосуществующие виды по каждому разрезу.

3. Составим матрицу отношений между видами. Для этого заполним заглавные части и диагональ матрицы кодовыми номерами всех видов, 


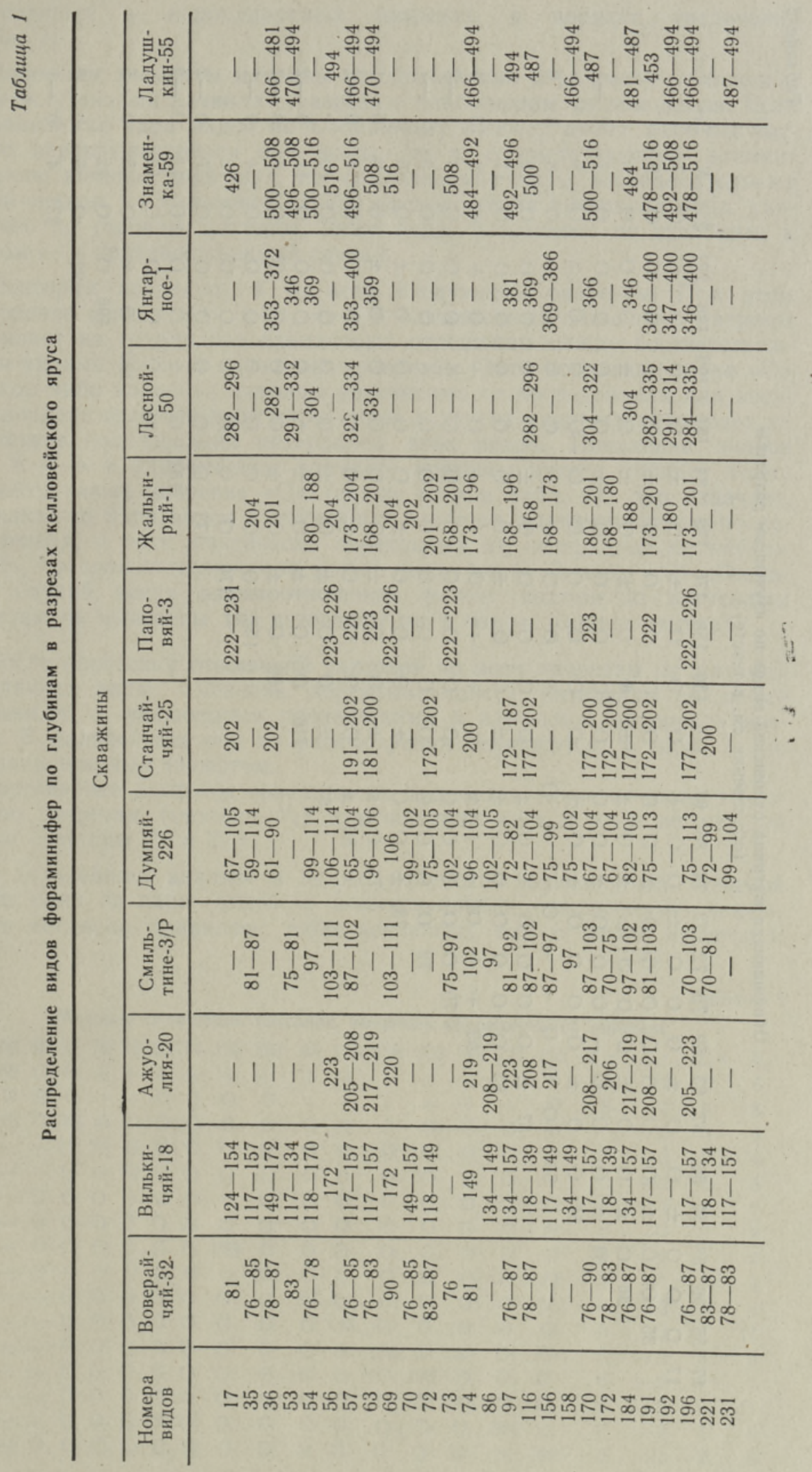




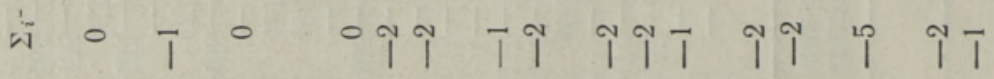

H -0 m Zทนのก  $\overrightarrow{\mathrm{N}}+1000+1+00+00+1+1000000000 \times 0$ 年 $\$ 00000000000000000000000 \%$ ब $0++100000+++00 \times 000000+10$ มี

\section{0+100000}

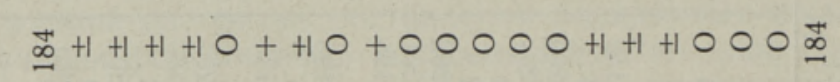

ก $00000+00+00+10+100000$ ก

I $+10+10000+10000+10+1+h 0 \frac{1}{10}$

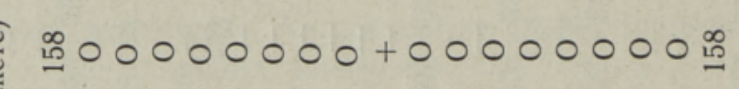

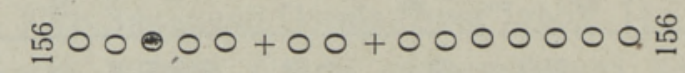

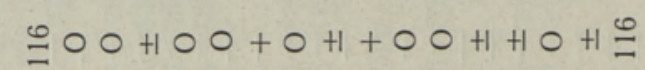

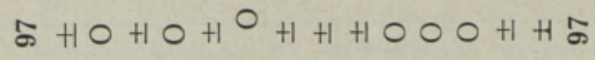

$\$ 00000+00+0000 \%$

ino hith ha hot ho hit

$i+10+100,00000 \%$

No0, $000,00+0$,

$500000+00+\frac{1}{16}$

$800010^{0} 008$

$z+10+10+10+18$

is $00000^{\circ}$ in

น 00000 i

เึ $+1+1+1+1$ 苗

ถ๊ 000 ஜ

๓००\%

냉요 낭

$\simeq$ 

(табл. 2).

4. Заполним элементы матрицы наблюдаемыми в разрезах отношениями между видами начиная с разреза с наибольшим числом видов. Если сравниваемая пара видов по выбранному разрезу имеет хотя бы частично перекрывающиеся интервалы, то в соответствующий элемент матрицы впишем знак «O». Из сравниваемой пары последовательных видов знаком «+» отметим случай расположения интервала вида, указанного по строкам матрицы, ниже вида, указанного по столбцам, а знаком «-» противоположный случай.

5. Повторим процедуру 4 по всем следующим разрезам. При этом повторение знаков «О», «+», «-»в одноименных элементах матрицы указывает на сохранение одинаковых отношений между соответствующими видами по всем изучаемым разрезам. Зато появление знака «O» в каком-либо элементе матрицы независимо от последования или сосуществования оравниваемой пары видов в других разрезах указывает на отсутствие последования интервалов этих видов. Появление знаков «+» и «-» в одноименном элементе матрицы вместе придает этому элементу также значение «О» (знаки «士» в табл. 2). Это означает, что какая-то пара видов в изученных разрезах противоположно последовательна, и хотя фактически перекрытия их интервалов не наблюдалось, предположительно оно может иметь место. Знаком «Х» обозначают случай, когда взаимоотношения между видами по изученным разрезам не выяснены (не встречены ни в одном разрезе вместе).

6. После нанесения отношений таксонов по всем разрезам на матрицу выявляются виды, имеющие перекрывающиеся интервалы со всеми другими, т. е. являющиеся проходящими в изученном интервале времени. В дальнейшем они из рассмотрения опускаются; в табл. 2 они показаны жирным шрифтом.

Составление матрицы завершается подсчетом встречаемости таксонов по разрезам (столбец $N_{i}$ ) и отношений «+» и «—» по каждой строке (столбцы $\Sigma_{i^{+}}$и $\Sigma_{i^{-}}$).

7. В дальнейшем анализе в целях получения однозначной искомой шкалы один из пары видов с невыясненными отношениями исключается согласно правилам: либо исключить вид с менее известным

Таблица 3

Матрица отношений таксонов, имеющих коррелятивное значение

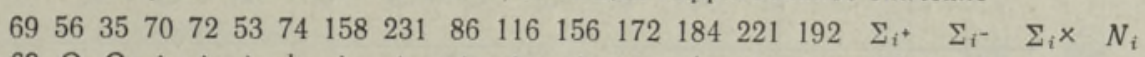
$69690 \mathrm{O}++++++++++++++130008$

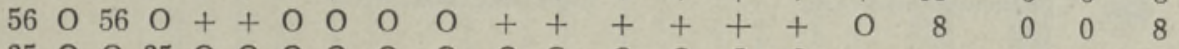

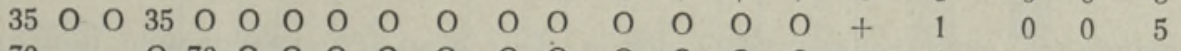
$70-\begin{array}{lllllllllllllllll} & 70 & \mathrm{O} & \mathrm{O} & \mathrm{O} & \mathrm{O} & \mathrm{O} & \mathrm{O} & \mathrm{O} & \mathrm{O} & \mathrm{O} & \mathrm{O} & \mathrm{O} & + & -2 & 0 & 4\end{array}$

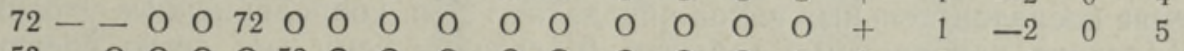
$\begin{array}{llllllllllllllllllll}53- & - & 0 & 0 & 0 & 53 & 0 & 0 & 0 & 0 & 0 & 0 & 0 & 0 & 0 & 0 & 0 & -1 & 0 & 7\end{array}$ $\begin{array}{llllllllllllllllllll}74-0 & 0 & 0 & 0 & 0 & 74 & 0 & 0 & 0 & 0 & 0 & 0 & 0 & 0 & 0 & 0 & -1 & 0 & 9\end{array}$ 158 - $\begin{array}{llllllllllllllllllll} & 0 & 0 & 0 & 0 & 0 & 0 & 158 & 0 & 0 & 0 & 0 & 0 & 0 & 0 & 0 & 0 & -1 & 0 & 4\end{array}$

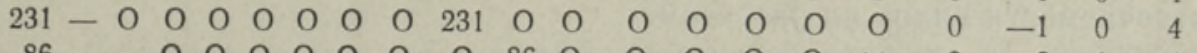

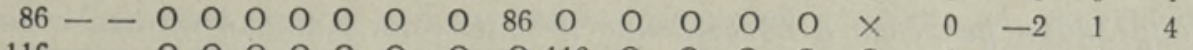

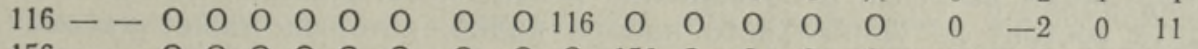

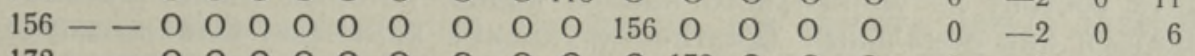
$\begin{array}{llllllllllllllllllll}172 & - & 0 & 0 & 0 & 0 & 0 & 0 & 0 & 0 & 0 & 0 & 172 & 0 & 0 & 0 & 0 & -2 & 0 & 7\end{array}$ $184-\begin{array}{lllllllllllllllllll}0 & \mathrm{O} & \mathrm{O} & \mathrm{O} & \mathrm{O} & \mathrm{O} & \mathrm{O} & \mathrm{O} & \mathrm{O} & \mathrm{O} & \mathrm{O} & 184 & \mathrm{O} & \mathrm{O} & 0 & -2 & 0 & 11\end{array}$ $\begin{array}{lllllllllllllllllllll}221 & - & 0 & 0 & 0 & 0 & 0 & 0 & 0 & 0 & 0 & 0 & 0 & 0 & 221 \times & 0 & -2 & 1 & 5\end{array}$

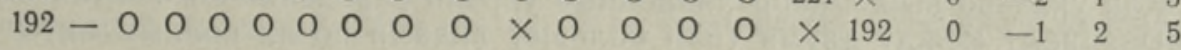


распространением, т. е. встреченный в меньшем числе разрезов, либо сохранить максимальное для корреляции число видов, либо постараться выполнить оба требования. В нашем материале, например, исключен вид 192, имеющий невыясненные отношения с видами 86 и 221 , встреченльми приблизительно с той же частотой в изученных разрезах.

8. Проверим непротиворечивость матрицы отношений (табл. 2). Для этого составим табл. 3, в ксторую выпишем все «непроходящие» виды (имеют хотя бы один знак «+» или «-») в порядке убывания количества знанов «-t» и увәличения количества знаков «-» по строкам. Тогда в упорядоғенной таким образом матрице все знаки «+» (по столбцам) должны быть выше знаков «O». В противном случае возникают противоречия при корреляции разрезов. Отметим, что вид 192 как раз имеет такое противоречие, что подтверждает правильность его исключения из дальнейшего анализа.

9. По оставшимся видам составим т. н. стандарт (табл. 4). В стандарте все сосуществующие во времени виды (связаны отношением «O») выписывают в одну строку, а последовательные - в разные строки. Сплошными линиями в стандарте выделено смыкание полных

Таблица 4

Фораминиферовый стандарт для изученных разрезов и стратиграфический возраст видов

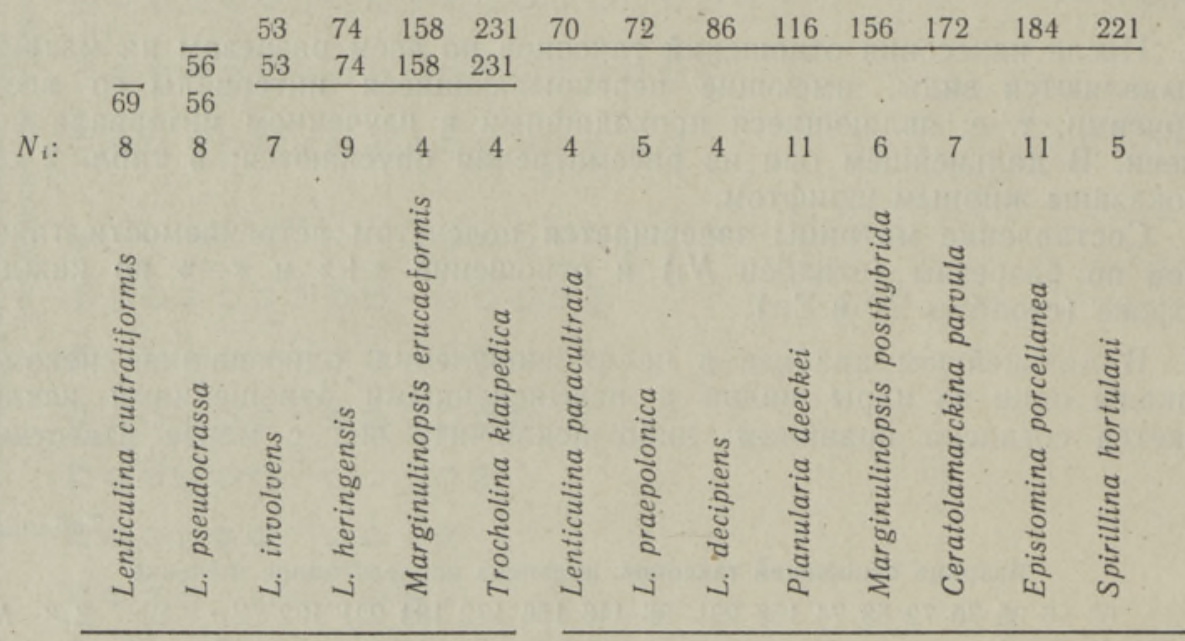

Среднекелловейские

Верхнекелловейские

интервалов последовательных видов. Тем самым исчезновение и появление последовательных видов определяют т. н. корреляционные уровни. Последние как сложные события (благодаря чему они легко идентифицируются в разрезах) образуют и линейную шкалу времени для данных разрезов. Самая нижняя строка в табл. 4 повторяет число встречаемости видов по разрезам.

Корреляция разрезов и обсуждение результатов. Каждая строка стандарта состоит из уникального набора видов, который может расоматриваться как зональный (диагностирующий) комплекс. Однако коррелящию разрезов при помощи стандарта проводят несколько иначе, чем по традиционному методу.

Как указано выше, корреляционные уровни определяются контактом (смыканием) между двумя (или больше) видами по их полным интер- 
валам существования согласно стандарту. В данном случае выявлены два таких уровня. Оказывается, что ниже первого (нижнего) корреляционного уровня остается только один среднекелловейский вид (с кодовым номером 69), а выше - часть средне- и позднекелловейских видов (начиная с номера 53 и правее по стандарту). Ниже второго (верхнего) корреляционного уровня остаются два среднекелловейских вида (69 и 56), а выше - все позднекелловейские виды (начиная с 70 и правее).' Тем самым, два древних уникальных набора состоят только из ореднекелловейских видов, часть из которых сосуществует и с позднекелловейскими $(53,74,158$ и 231$)$, и младший уникальный набор только из позднекелловейских видов. Это означает, что границу между средним и верхним келловеем следует искать там, где в коррелируемых разрезах проходит смыкание интервалов видов древнего и младшего наборов.

Положение корреляционных уровней, т. е. искомой границы, в коррелируемых разрезах определяется находками соответствующих «стандартных» видов. Так, нижний уровень определяется исчезновением (последними находками) вида под номером 69 и появлением (первой находкой) одного из видов под номерами $53,74,158,231$; верхний соответственно исчезновением номера 56 и появлением одного из номеров $170,72,86,116,156,172,184,221$. Так как находки видов в разрезах обычно не совпадают с интервалом их существования, то для каждого корреляционного уровня трудно выявить одну точку в разрезе, можно установить лишь т. н. интервал неопределимости, в пределах которого искомая граница должна находится. Найденные пределы этих корреляционных уровней показаны цифрами (глубинами на корреляционной схеме).

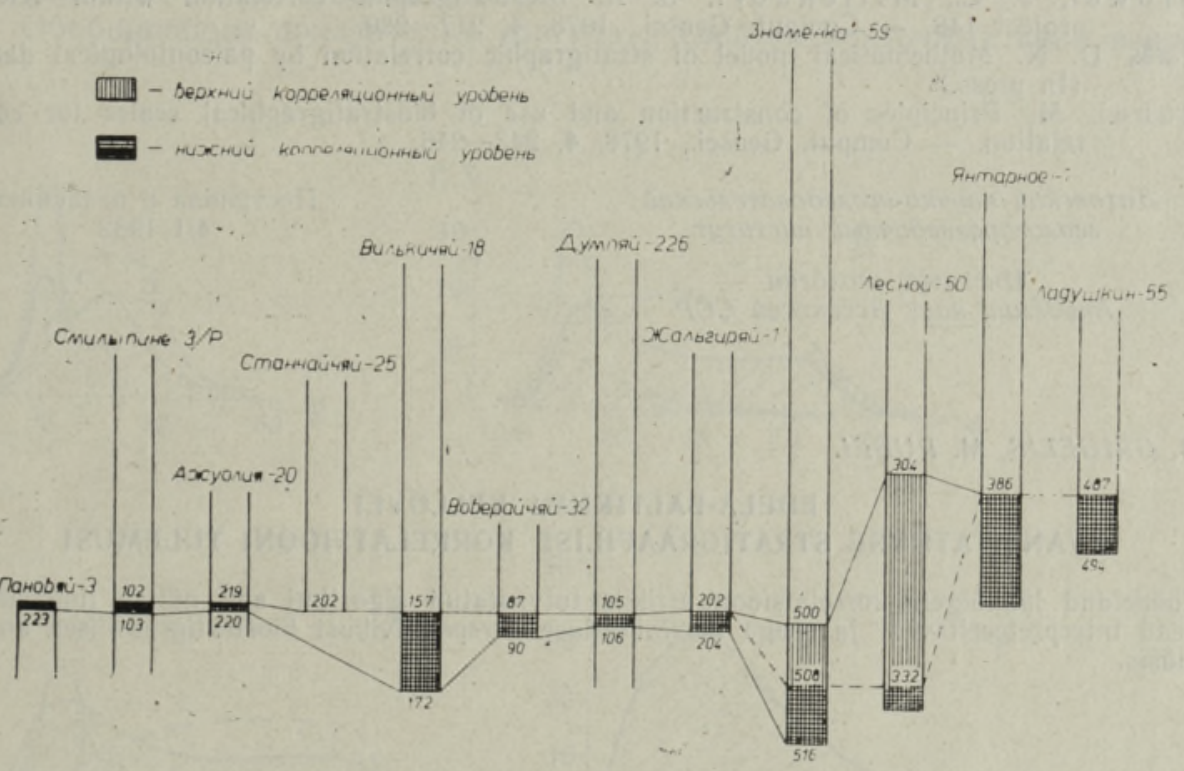

Согласно этому. в большинстве разрезов (точнее, за иоключением скважин Лесной-50 и Знаменка-59) оба выявленных корреляционных уровня совпали полностью. Тем самым, все разрезы можно датировать относительно найденной фораминиферовой шкалы по трем следующим интервалам: 1 - интервалы, оставшиеся ниже обеих корреляционных уровней (точнее, нижнего предела нижнего уровня) — несомненный средний келловей (или ниже), 2 - суммарные интервалы неопредели- 
мости по обеим корреляционным уровням - возраст по изученным данным точно не идентифицируется; либо средний, либо верхний келловей и 3 - интервалы, оставшиеся выше обеих корреляционных уровней (точнее, выше верхнего предела верхнего уровня) - несомненный верхний келловей (или выше).

Используя зональную терминологию, для интервалов 1 руководящими (зональными) являются виды под номерами 69 и 56, а для интервалов 3 - виды под номером 70 и от него правее по стандарту. Вывод о зональности видов полностью согласуется с данными биостратиграфических исследований (Григялис, 1980).

Bыводы. Полученные результаты показывают работоспособность рассмотренного алгоритма при анализе микропалеонтологического материала с непрерывным распределением видов в отложениях морских разрезов. Он дает возможность описать ход классического биостратиграфического анализа. Вместе с тем полученный опыт указывает на необходимость и целесообразность привлечения бо́льшего числа данных (по количеству видов, по стратиграфическим интервалам и по количеству разрезов), чему способствует наличие машинных программ обработки на основе указанного алгоритма.

\section{Л ИТ Е РА Т Р Р А}

Григялис А. А. Балтийская юра (фораминиферы и зональная стратиграфия). Автореф. докт. дис. Л., 1980.

Оле йни ко в А. Н., П а е в к а я Е. Б. Применение количественных методов стратиграфической корреляции в СССР. (В печати).

Рубель М. П. К биологической конструкщии времени в геологии. - Изв. АН ЭстССР. Хим. Геол., 1976, 25, 136-144.

Brower, J. C., Millendorf, S. A. Biostratigraphic correlation within IGCP project 148. - Comput. Geosci., 1978, 4, 217-220.

Pak, D. N. Mathematical model of stratigraphic correlation by paleontological data. (In press.)

Rubel, M. Principles of construction and use of biostratigraphical scales for correlation. - Comput. Geosci., 1978, 4, 243-246.

Литовский научно-исследовательский геологоразведочный институт

Поступила в редакцию

Институт геологии

Академии наук Эстонской ССР

A. GRIGELIS, M. RUBEL

\section{EDELA-BALTIKUMI KELLOVEI KVANTITATIIVSE STRATIGRAAFILISE KORRELATSIOONI TULEMUSI}

Töödeldud läbilōigete korrelatsioon artiklis tutvustatud algoritmi abil näitab tulemuste head interpreteeritavust ja seega masintöötluse perspektiivikust biostratigraafilises analüüsis.

A. GRIGELIS, M. RUBEL

\section{RESULTS OF THE QUANTITATIVE STRATIGRAPHIC CORRELATION OF THE CALLOVIAN IN THE SOUTH-WEST BALTIC}

The distributional data of 26 species of foraminifers from 12 Callovian sections in the South-West Baltic are summarized into a composite standard according to the algorithm introduced by the author. The correlation of the sections studied by means of such a standard coincides well with previous results. It is concluded that computer processing of a copious set of data will prove helpful and desirable. 